\section{Phase-III-Studie mit Nedaplatin bei NSCLC}

\begin{abstract}
Beim Lungenkarzinom sind neue Therapeutika nötig, vor allem gilt dies für das fortgeschrittene, nichtkleinzellige Lungenkarzinom (NSCLC) vom Plattenepithel-Typ. In einer randomisierten Phase-III-Studie haben japanische Ärzte das neue Cisplatin-Derivat Nedaplatin getestet.
\end{abstract}

Bim eim fortgeschrittenen Plattenepithelkarzinom der Lunge greift man bisher auf platinbasierte Chemotherapien zurück. Dabei könnte das neue Cisplatin-Derivat Nedaplatin einen Fortschritt bedeuten, das weniger neurotoxisch ist und gastrointestinale Nebenwirkungen verursachen soll. In Phase-I-Studien führte es vor allem beim Plattenepithelkarzinom zu günstigen Tumorantworten; dosislimitierend waren Neutropenie und Thrombozytopenie. Auch eine Phase-I/II-Studie zeigte beim rezidivierten oder fortgeschrittenen Plattenepithelkarzinom eine vielversprechende Aktivität. Aber wie sieht es mit dem Überleben aus? Um diese Frage zu beantworten, verglichen japanische Kollegen in einer Phase-III-Studie Nedaplatin und Cisplatin, jeweils mit Docetaxel gegeben, bei 355 Patienten mit Plattenepithelkarzinom der Lunge im Stadium IIIB/IV oder mit einem postoperativ aufgetretenen Rezidiv und einem ECOG-Performancestatus von 0 oder 1. Die Patienten erhielten intravenös Docetaxel $\left(60 \mathrm{mg} / \mathrm{m}^{2}\right)$ und entweder $100 \mathrm{mg} /$ $\mathrm{m}^{2}$ Nedaplatin oder $80 \mathrm{mg} / \mathrm{m}^{2}$ Cisplatin alle 3 Wochen für 4-6 Zyklen. Primärer Endpunkt war das Gesamtüberleben.

349 Patienten wurden in die modifizierte Intention-to-Treat-Analyse aufgenommen, 177 aus der Nedaplatin- und 172 aus der Cisplatin-Gruppe. Das Gesamtüberleben war unter Nedaplatin signifikant länger als unter Cisplatin (median 13,6 vs. 11,4 Monate; Hazard Ratio [HR] 0,81; $\mathrm{p}=0,037$ ).
Nausea vom Grad $\geq 3$ (7 von 177 Patienten in der Nedaplatin- und 25 von 175 in der Cisplatin-Gruppe), Fatigue (6 vs. 20), Hyponatriämie (24 vs. 53) und Hypokaliämie (4 vs. 15) waren häufiger unter Cisplatin, während Leukopenien vom Schweregrad $\geq 3$ (98 vs. 77), Neutropenien (146 vs. 123) und Thrombozyptopenien (16 vs. 0) häufiger unter $\mathrm{Ne}$ daplatin auftraten. Im Nedaplatin-Arm gab es 4 behandlungsbedingte Todesfälle, unter Cisplatin 3.

Fazit: Das Gesamtüberleben war signifikant länger unter Nedaplatin als unter Cisplatin; die Regimes hatten in der japanischen Population unterschiedliche Sicherheitsprofile. Nedaplatin plus Docetaxel könnte demnach eine neue Option für Patienten mit fortgeschrittenem oder rezidiviertem Plattenepithelkarzinom sein.

Christina Berndt

Shukuya T et al. Nedaplatin plus docetaxel versus cisplatin plus docetaxel for advanced or relapsed squamous cell carcinoma of the lung (WJOG5208L): a randomised, open-label, phase 3 trial. Lancet Oncol. 2015;16(16):1630-8.

\title{
Option für Ältere mit einem Kleinzeller
}

\section{Standardbehandlung beim kleinzelligen Lungenkarzinom (SCLC) ist die kombinierte Strahlenchemotherapie. Eine retrospektive US-Studie sollte klären helfen, ob ältere Patienten mehr von der Kombination profitieren als von einer alleinigen Chemotherapie.}

$E_{s}^{\text {tom }}$ wa ein Drittel der Patienten mit SCLC befindet sich bei Erstdiagnose im Stadium „Limited Disease“. Standard ist hier die kombinierte Strahlenchemotherapie mit 5-Jahres-Überlebensraten zwischen 20 und $25 \%$. Fast jeder zweite Patient ist älter als 70, etwa $10 \%$ sind älter als 80 Jahre. Daran erinnern US-Onkologen um Christopher D. Corso. Da es keine randomisierten Studien zum Vergleich der beiden Therapiemodalitäten bei Älteren gibt, durchforsteten sie retrospektiv die "National Cancer Data Base“, die ihre Informationen von mehr als 1.500 US-Kliniken bezieht.

Für die Auswertung standen die Befunde von jeweils über 3.000 Patienten zur Verfügung, die zwischen 2003-2011 entweder nur eine Chemotherapie oder eine Kombination aus Chemotherapie und Bestrahlung erhalten hatten. Die Patienten waren mindestens 70 Jahre alt und hatten ein SCLC im Stadium I-III.

Wie Corso und Kollegen berichten, lag der Anteil der Patienten, die nach 3 Jahren noch lebten, nach zusätzlicher Bestrahlung um 15,7\% absolut höher als nach alleiniger Chemotherapie (22 vs. 6,3\%). Auch beim medianen Gesamtüberleben schnitten Patienten mit der Kombinationstherapie besser ab (15,6 vs. 9,3 Monate). In der Gruppe mit Bestrahlung lagen die Überlebenschancen um $48 \%$ höher als in der Vergleichsgruppe, wie aus der multivariaten Analyse hervorging (Hazard Ratio [HR] 0,52, $95 \%$-Konfidenzintervall $0,49-0,54$; $\mathrm{p}<0,001)$.
Die meisten Patienten hatten zur Chemotherapie mindestens 2 Präparate erhalten, wobei der Anteil in der Chemoradiotherapie-Gruppe höher lag (91,2 vs. $86,4 \%)$. Im Median betrug die verabreichte Strahlendosis knapp 60 Gy (mindestens 50,4 und maximal 61,2 Gy). Patienten, die gleichzeitig bestrahlt und chemotherapiert worden waren, hatten nach 3 Jahren etwas höhere Überlebensraten als Patienten mit einer sequenziellen Therapie (24,2 vs. $20,3 \%)$.

Fazit: Ältere Patienten mit einem auf den initialen Hemithorax begrenzten SCLC („Limited Disease“) profitieren Studiendaten zufolge mehr von einer modernen Chemoradiotherapie als von einer Chemotherapie allein - vorausgesetzt, sie vertragen die Kombination. Den meisten Nutzen haben die Betroffenen offenbar von einer gleichzeitigen Strategie.

Peter Leiner

Corso CD et al. Role of Chemoradiotherapy in EIderly Patients With Limited-Stage Small-Cell Lung Cancer. J Clin Oncol. 2015;33(36):4240-6. 